\title{
Effect of curvature on structures and vibrations of zigzag carbon nanotubes: A first-principles study
}

\author{
MOUSUMI UPADHYAY KAHALY* and UMESH V WAGHMARE \\ Theoretical Sciences Unit, Jawaharlal Nehru Centre for Advanced Scientific Research, \\ Bangalore 560 064, India
}

\begin{abstract}
First-principles pseudopotential-based density functional theory calculations of atomic and electronic structures, full phonon dispersions and thermal properties of zigzag single wall carbon nanotubes (SWCNTs) are presented. By determining the correlation between vibrational modes of a graphene sheet and of the nanotube, we understand how rolling of the sheet results in mixing between modes and changes in vibrational spectrum of graphene. We find that the radial breathing mode softens with decreasing curvature. We estimate thermal expansion coefficient of nanotubes within a quasiharmonic approximation and identify the modes that dominate thermal expansion of some of these SWCNTs both at low and high temperatures.
\end{abstract}

Keywords. Carbon nanotubes; first-principles study.

\section{Introduction}

The great interest in one-dimensional nanostructures such as nanotubes and nanowires is partly driven by the possibility of their being used as active components in nanosized devices. Carbon nanotubes (CNTs), discovered in 1991 by Ijima, are considered to be a very important and interesting class of nanostructures due to their unique electrical, mechanical and thermal properties, and possibility of their industrial applications. A single wall carbon nanotube (SWCNT) is formed by rolling a two-dimensional graphene sheet, which has a planar crystalline structure made with hexagonal carbon rings. A multiwall nanotube is made up of more than one SWCNTs of different diameters which share a common axis. Understanding the properties of individual SWCNTs and the interaction between them is important to understand the properties of multiwall CNTs as well as SWNTs in a bundled form. Diameter of SWCNTs is typically of the order of a few nanometers and their length typically exceed a micrometer.

Nanotubes are described by chiral vector, $\vec{C}_{\mathrm{h}}$, that connects two crystallographically equivalent sites on the unrolled graphene sheet. $\vec{C}_{\mathrm{h}}=n \times \vec{a}_{1}+m \times \vec{a}_{2}$, where $n$, $m$ are integers and $\vec{a}_{1}, \vec{a}_{2}$ are unit vectors of honeycomb graphene lattice. Thus, each SWCNT is uniquely labeled with a pair of integers $(n, m)$ describing its chirality. ( $n$, $n$ ) CNTs are called as 'armchair' nanotubes and $(n, 0)$ CNTs are called as 'zigzag' nanotubes. Inspite of the fact that armchair nanotubes are more readily formed (Thess et al 1996) in comparison to zigzag nanotubes using different experimental techniques like chemical vapour

\footnotetext{
*Author for correspondence (mousumi@jncasr.ac.in)
}

deposition, laser ablation etc, the zigzag nanotubes are also thermodynamically very stable. Moreover, metal-tosemiconductor transitions can be induced more readily in metallic zigzag nanotubes than in armchair nanotubes ( $\mathrm{Lu}$ et al 2005). Thus it is important to understand the energetics, stability and size-dependent physical properties of these nanotubes.

Earlier, first principles calculations (Cabria et al 2003) of the electronic structures of narrow zigzag CNTs revealed that tubes with diameter $<0.4 \mathrm{~nm}$ are unstable. Growth (Bulusheva et al 1998) and transport in oxygendimer-doped zigzag carbon nanotubes (Jhi et al 2005) have been studied within $a b$ initio pseudopotential density functional method. However, effect of curvature and inter-tube interaction on the physical properties of zigzag CNTs deserves to be understood in detail. In this work, we use first-principles calculations to determine atomic structures, electronic properties and vibrational spectra of zigzag CNTs $((n, 0)$, with $n=3$ to 23$)$ in isolated and bundled forms. This paper is structured as follows: In $\S 2$, we describe details of the density functional theory (DFT) based methods used. In $\$ 3$, we present a comparative study of lattice parameters, atomic structures and cohesive energies of zigzag SWCNTs with different chiralities (for $n=3$ to $n=23$ ) and discuss their electronic properties. We present results of their vibrational spectra in $\S 4$ and finally summarize in $\S 5$.

\section{Methods}

We use SIESTA (Ordejon et al 1996; Soler et al 2002) implementation of the density functional theory, which employs a localized orbital basis. While it can be used in the linear scaling mode, we used an option of explicit 
diagonalization of the hamiltonian as the size of our systems is moderate. We used soft norm-conserving pseudopotentials (Troullier and Martins 1991) and CeperleyAlder exchange correlation energy within a local density approximation (LDA). Mesh used in our calculations with localized basis has a resolution corresponding to a planewave energy cutoff of $200 \mathrm{Ry}$. Periodic boundary conditions are used with a supercell that has the periodicity of CNT along $z$-axis and its size in the $a b$ plane is much larger than the diameter of the CNT to ensure negligible interaction among its periodic images (with a vacuum of about $15 \AA$ separating them). Resulting Brillouin zones (BZ) have a very small dimension in the $a b$ plane and is sampled with a Monkhorst-Pack (1976) meshes, $1 \times 1 \times 33$ (treating $17 k$ points) for integrations. For SWCNTs in bundled form, we optimized the hexagonal lattice of CNTs and sampled its Brillouin zone with a $3 \times 3 \times 33 k$ point mesh. For all systems of study, geometry optimization was carried out until all the forces acting on all atoms decreased below $0.03 \mathrm{eV} / \AA^{-1}$.

We use a frozen-phonon method to calculate phonons of a CNT, and use a supercell consisting of 5 unit cells stacked along $z$-axis. Phonon dispersion for each $(n, 0)$ SWCNT was determined along the high symmetry direction $\Gamma(0,0,0)$ to $A(0,0, \pi / c)$. To estimate the thermal expansion of $\mathrm{CNT}$, we calculate full phonon dispersions in three configurations: (a) equilibrium ( $c=c_{0}, c_{0}$ being the optimal $c)$, (b) elongated $\left(c=1 \cdot 01 c_{0}\right)$, and (c) contracted $\left(c=0.99 c_{0}\right)$. For each configuration (given by $\left.c\right)$, we relaxed the structure, and obtained real-space force constant matrices through frozen phonon calculations. We use VIBRA package (available with SIESTA) for (a) using the real-space force constants to obtain dynamical matrices at $q$-points on a fine mesh of 61 points in the BZ, and (b) diagonalization of the dynamical matrices. This procedure yields phonon dispersion and density of phonon states (ph-DOS) for each of the configurations, which are subsequently used in determining derivatives of phonon frequencies with $c$ using a finite difference formula.

\section{Properties of SWCNTs in isolated and bundled forms}

The geometrical parameters e.g. the diameter $\left(d_{0}=2 r_{0}\right)$, chiral angle $\left(\theta_{0}\right)$, lattice constant $\left(c_{0}\right)$ etc ideally can be expressed as functions of chirality $(n, m)$, such that

$$
d_{0}=a \sqrt{\left(n^{2}+n m+m^{2}\right)} / \pi
$$

where $a=l_{\mathrm{B}} \times \sqrt{3}, l_{\mathrm{B}}$ being interatomic spacing in graphene.

$$
\begin{aligned}
& \cos \theta_{0}=(2 n+m) / 2 \sqrt{n^{2}+n m+m^{2}}, \\
& c_{0}=3 r_{0} \sqrt{3\left(n^{2}+n m+m^{2}\right)} / \operatorname{GCD}(2 n+m, 2 m+n),
\end{aligned}
$$

GCD being greatest common divisor of the two integers.

For zigzag nanotube, $m=0$. Formulae (1), (2) and (3) are valid under the assumption that there is no effect of curvature on the structure of SWCNTs due to rolling of the continuous graphene sheet. However, in reality, most bonds in a SWCNT get directly affected by the effect of curvature. This effect is found to be very prominent for nanotubes with small diameter due to their greater curvature. Effects of curvature on properties partly contribute to their nano-size dependence.

\subsection{Stability, atomic and electronic structures}

We study SWCNTs of different radii in two forms: (i) isolated and (ii) bundled, both of which are realized in experiments. Results for structural and geometrical properties of different zigzag SWCNTs suggest that though the lattice constant, $c$, in its two forms is the same, each SWCNT in a bundle has a smaller radius than that in its isolated form (see table 1, columns 3 and 4). However, this difference diminishes as the diameter of the tube increases or the curvature reduces. Different bonds in the SWCNTs are found to deviate from their ideal values of $1.42 \AA$ in the unrolled graphene sheet (see table 1, column 5). In a $(n, 0)$ zigzag nanotube, two sides of each hexagon are parallel to the tube axis. These two bonds (first and third numbers in column 5 of table 1) in each carbon ring are least affected due to effect of curvature upon rolling. Change in curvature thus affects the $\mathrm{C}-\mathrm{C}$ bond-strength and hence the vibrational spectra which are responsible for many physical properties like the thermal expansion coefficient (TEC), $\alpha$ or specific heat, $C_{\mathrm{p}}$.

Our results for cohesive energy, $E_{\mathrm{c}}$, per atom shows that $E_{\mathrm{c}}$ of a nanotube in the bundled form is more than that in the isolated form (see table 1, column 6). Due to interactions with neighbouring tubes in the bundle form, there is additional cohesion resulting in its slightly higher stability, consistent with their greater abundance in experiments. Cohesive energy of all SWCNTs increases with increasing diameter or chirality index, $n$. For example, for SWCNT in isolated form, monotonic increase in $E_{\mathrm{c}}$ as a function of diameter (see figure la) can be described by a non-linear function form (shown by solid line in the fit)

$$
A+B d+C d \times \exp (-d)
$$

where $A, E, C$ are constants and $d$ the diameter of $(n, 0)$ zigzag nanotubes.

As is well known (Hamada et al 1992), the electronic structure of a SWCNT depends on the divisibility of its chirality index, $n$, by 3 , i.e. number of bonds shared by each atom in the hexagonal network. Thus, $(3 N, 0)$ nanotubes are expected to be metallic, and $(3 N \pm 1,0)$ are expected to be semiconducting. Our results of electronic structures of zigzag nanotubes also follow the same trend, except for nanotubes with very small diameter. As shown 
Table 1. Comparison of the values of the lattice constant, $c$, along the axis of SWCNT, diameter, $d$, bond lengths, $l_{\mathrm{B}}$, cohesive energy, $E_{\mathrm{c}}$ per atom $(\mathrm{eV} /$ atom $)$ and bandgap, $E_{\mathrm{g}}(\mathrm{eV})$ for zigzag SWCNTs with different chirality index, $n$.

\begin{tabular}{|c|c|c|c|c|c|c|}
\hline System & Form & $c(\AA)$ & $d(\AA)$ & $l_{\mathrm{B}}(\AA)$ & $E_{\mathrm{c}}(\mathrm{eV} /$ atom $)$ & $E_{\mathrm{g}}(\mathrm{eV})$ \\
\hline \multirow[t]{2}{*}{$(3,0)$} & isolated & $4 \cdot 235$ & $2 \cdot 67$ & $1.409,1.507,1.409,1.507$ & 8.43 & $0 \cdot 0$ \\
\hline & bundled & $4 \cdot 235$ & $2 \cdot 38$ & $1.411,1.493,1.412,1.489$ & $8 \cdot 71$ & $0 \cdot 0$ \\
\hline \multirow[t]{2}{*}{$(4,0)$} & isolated & $4 \cdot 244$ & $3 \cdot 41$ & $1.410,1.488,1.411,1.489$ & $8 \cdot 92$ & $0 \cdot 0$ \\
\hline & bundled & $4 \cdot 244$ & $3 \cdot 20$ & $1.412,1.473,1.413,1.469$ & $9 \cdot 03$ & $0 \cdot 0$ \\
\hline \multirow[t]{2}{*}{$(5,0)$} & isolated & $4 \cdot 269$ & $4 \cdot 22$ & $1.415,1.465,1.418,1.462$ & $9 \cdot 18$ & $0 \cdot 0$ \\
\hline & bundled & $4 \cdot 269$ & $4 \cdot 03$ & $1.416,1.449,1.417,1.449$ & $9 \cdot 32$ & $0 \cdot 0$ \\
\hline \multirow[t]{2}{*}{$(6,0)$} & isolated & $4 \cdot 272$ & 4.89 & $1.418,1.453,1.419,1.453$ & $9 \cdot 34$ & $0 \cdot 0$ \\
\hline & bundled & $4 \cdot 272$ & 4.75 & $1.417,1.443,1.418,1.445$ & $9 \cdot 51$ & $0 \cdot 0$ \\
\hline \multirow[t]{2}{*}{$(7,0)$} & isolated & $4 \cdot 281$ & $5 \cdot 60$ & $1.424,1.444,1.425,1.443$ & $9 \cdot 44$ & $0 \cdot 12$ \\
\hline & bundled & $4 \cdot 281$ & $5 \cdot 49$ & $1.423,1.437,1.424,1.436$ & $9 \cdot 55$ & $0 \cdot 10$ \\
\hline \multirow[t]{2}{*}{$(8,0)$} & isolated & $4 \cdot 286$ & $6 \cdot 41$ & $1.426,1.442,1.426,1.442$ & $9 \cdot 50$ & $0 \cdot 46$ \\
\hline & bundled & $4 \cdot 286$ & $6 \cdot 31$ & $1.424,1.436,1.423,1.436$ & $9 \cdot 57$ & 0.43 \\
\hline \multirow[t]{2}{*}{$(9,0)$} & isolated & $4 \cdot 288$ & $7 \cdot 14$ & $1.427,1.440,1.427,1.440$ & $9 \cdot 55$ & $0 \cdot 0$ \\
\hline & bundled & $4 \cdot 288$ & $7 \cdot 06$ & $1.424,1.433,1.425,1.435$ & $9 \cdot 59$ & $0 \cdot 0$ \\
\hline \multirow[t]{2}{*}{$(10,0)$} & isolated & $4 \cdot 290$ & $7 \cdot 90$ & $1.429,1.437,1.430,1.437$ & 9.58 & 0.47 \\
\hline & bundled & $4 \cdot 290$ & $7 \cdot 85$ & $1.427,1.433,1.427,1.432$ & $9 \cdot 61$ & 0.41 \\
\hline \multirow[t]{2}{*}{$(12,0)$} & isolated & $4 \cdot 291$ & $9 \cdot 49$ & $1.429,1: 435,1.429,1.435$ & $9 \cdot 62$ & $0 \cdot 0$ \\
\hline & bundled & $4 \cdot 291$ & $9 \cdot 45$ & $1.427,1.432,1.426,1.433$ & $9 \cdot 64$ & $0 \cdot 0$ \\
\hline \multirow[t]{2}{*}{$(14,0)$} & isolated & $4 \cdot 291$ & 11.09 & $1.429,1.434,1.429,1.434$ & $9 \cdot 65$ & $0 \cdot 52$ \\
\hline & bundled & $4 \cdot 291$ & $11 \cdot 08$ & $1.427,1.433,1.428,1.434$ & $9 \cdot 66$ & 0.45 \\
\hline \multirow[t]{2}{*}{$(23,0)$} & isolated & $4 \cdot 291$ & $18 \cdot 17$ & $1.430,1.432,1.430,1.432$ & $9 \cdot 69$ & 0.46 \\
\hline & bundled & $4 \cdot 291$ & $18 \cdot 15$ & $1.429,1.432,1.430,1.431$ & $9 \cdot 70$ & 0.43 \\
\hline
\end{tabular}

in figure $1 b$, the comparison between the electronic density of states (DOS) of the $(10,0)$ SWCNT and $(12,0)$ SWCNT indicates that $(10,0)$ SWCNT is semiconducting with a bandgap of about $0.5 \mathrm{eV}$ whereas $(12,0)$ SWCNT is metallic. Secondly, in contrast to the prediction using tight binding approximations but in agreement with the experimental results (Odom et al 2000), the DOS of each of these nanotubes (with diameter $<10 \AA$ ) is asymmetric outside the energy interval of $\pm 1 \mathrm{eV}$ about the Fermi level. This reflects on the interactions among electronic states with energies close to the Fermi energy. As the diameter of the nanotube increases, the DOS of a nanotube becomes increasingly symmetric about the Fermi level. However, the expected semiconducting bandgap closes down to zero even in $(3 N \pm 1,0)$ zigzag nanotubes (where $N=$ integer) with diameter $<0.5 \mathrm{~nm}$, due to the influence of extreme curvature of their walls on the oneelectron spectrum of the $\pi$ electrons (see table 1, column 7). When a graphene sheet is rolled into a nanotube, it changes the hybridization of the carbon atoms. In graphite, $\pi$ orbitals are represented by the lone $2 p$ carbon states normal to the graphene plane, and $\sigma$ orbitals are linear combinations of in-plane $2 s$ and $2 p$ atomic orbitals. In carbon nanotubes, the radial orbitals, with a radial node in the cylinder, are analogous to the lone $\pi$ orbitals of graphite and, yet contain an admixture of $2 s$ carbon atomic orbitals; this changes the character of the electronic structure of carbon nanotubes, specially those with small diameters. We further note that the bandgap of a semiconducting nanotube is always less in its bundled form than that in the isolated form (table 1, column 7).

\section{Vibrational properties}

Analysis of different phonon modes using Raman spectroscopy of SWCNTs is useful in characterization of SWNTs and understanding their properties. We have determined full phonon dispersions for isolated zigzag SWCNTs as a function of $\vec{k}=(0,0, k) \pi / c$ (parallel to the SWCNT-axis). However, we present them here in the context of $(7,0)$ SWCNT (diameter, $\simeq 5.6 \AA$ ), as shown in figure 2. Zone-centre phonons have wider range in frequency than the zone-boundary phonons. We particularly point out the presence of branches at low frequencies that exhibit a dispersion that is quadratic in $k$; they play an important role in determining thermal properties of the nanotubes. Frequency of these branches at $\Gamma$ point decreases with $n$.

We focus on two distinct types of Raman active modes for each of the nanotubes (isolated form): (i) radial breathing mode (RBM) where all atoms in the SWCNT move in phase along the radial direction, and (ii) G-band modes with atomic displacements tangential to the surface of the tube (see figures 3(d), (e) and (f) for visual description of the eigen-displacements corresponding to these modes). The former has a vanishing frequency for graphene sheet and the latter (G-band) corresponds to its in-plane optical vibrational mode, which is doubly degenerate. It splits in the case of a nanotube into modes with atomic displacement perpendicular (GB2 mode) and parallel (GB1 mode) to the $c$-axis with lower and higher frequencies, respectively. We show the variation of the frequencies of these three modes at $\Gamma$ point for $(n, 0)$ zigzag nanotubes, with $n$ 
varying from 4 to 23 (see figures 3(a), (b) and (c) for RBM, GB2 and GB1, respectively) at the $\Gamma$ point, $k=$ $(0,0,0)$. From continuum elastic theory (Kurti et al 1998; Saito et al 1998; Sanchez-Portal et al 1999), it is readily shown that the frequency of the RBM $\left(\omega_{\mathrm{RBM}}\right)$ scales inversely with the diameter of the tube: $\omega_{\mathrm{RBM}} \propto 1 / d$ (shown by black solid line in figure 3(a)). Frequencies of RBM are found to be more strongly dependent on the diameter (i.e. curvature of the tube) than those of the G-band modes. GB2 modes show stronger curvature dependence than $\mathrm{GBl}$ modes. As $n$ is increased, $\omega_{\mathrm{GB} 1}$ and $\omega_{\mathrm{GB} 2}$ converge to the graphite's highest vibrational frequencies, $\omega_{\mathrm{G} 0} \sim 1695 \mathrm{~cm}^{-1}$. With increase in the tube diameter, effects of curvature decrease and $\omega_{\mathrm{RBM}}$ gradually decreases as $1 / d$ to zero.

Another way of understanding the effects of curvature on phonon dispersion of SWCNTs is developed through the correlation of its normal modes with that of an unrolled graphene sheet. This correlation function is defined by

$$
C_{i, j}\left(\omega_{\mathrm{N}}, \omega_{\mathrm{S}}^{\prime}\right)=\sum_{i, j} \delta\left(\omega-\omega_{i \mathrm{~N}}\right) \delta\left(\omega-\omega_{j \mathrm{~S}}^{\prime}\right)\left|\left\langle e_{i \mathrm{~N}} \mid e_{j \mathrm{~S}}\right\rangle^{2}\right|,
$$

where $\omega_{i \mathrm{~N}}$ and $\omega_{j \mathrm{~S}}$ imply different $i$ th phonon mode of the nanotube and $j$ th phonon mode of the graphene sheet. In
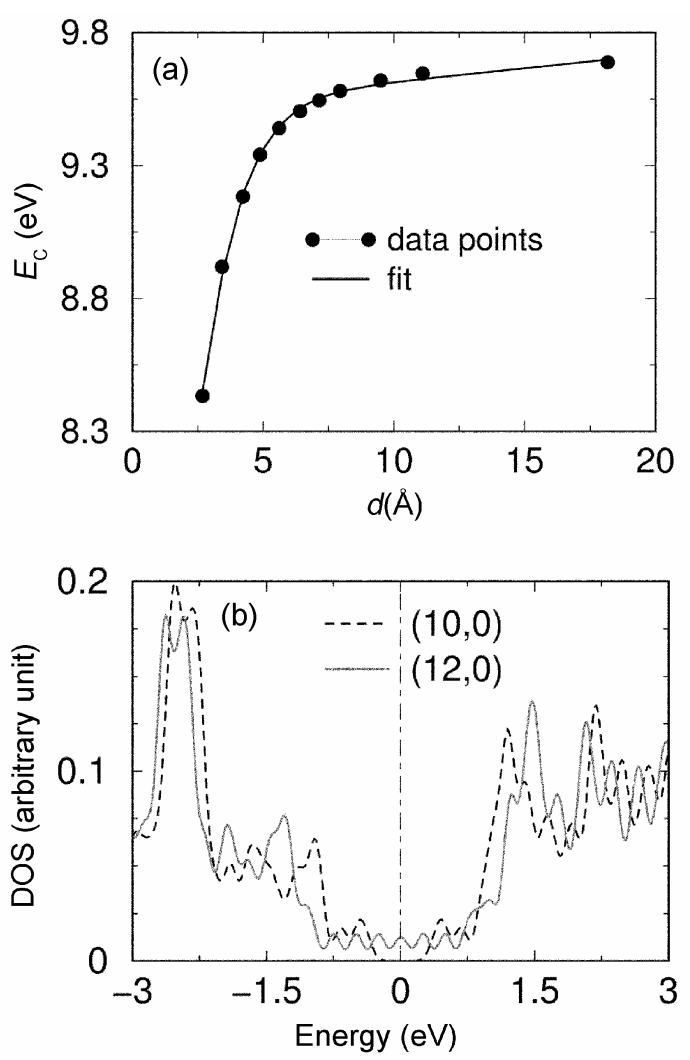

Figure 1. (a) Cohesive energy of isolated zigzag SWCNT as a function of diameter and (b) electronic densities of states of $(10,0)$ semiconducting (dashed line) and $(12,0)$ metallic SWCNT (solid line) the 3-dimensional plot (see figure 4 ) of $C_{i, j}$ of $(8,0)$ SWCNT, the presence of many off-diagonal small kinks reflects the mixing between different modes of graphene sheet to yield the normal modes of SWCNT. Clearly, as shown in figure 4 , the low frequency modes of the graphene sheet mix strongly and are modified upon rolling it into a SWCNT. If there were no mixing among the modes, the $C_{i, j}$ would take a diagonal form and the picture would have a single wall-like feature of height, 1 , along the diagonal. We also find such mixing is weaker for SWCNTs with larger diameter (not shown here).

\subsection{Thermal properties}

Access to the full phonon dispersion of a SWCNT allows us to determine some of its thermal properties (like the thermal expansion coefficient, $\alpha$ and specific heat, $C_{\mathrm{p}}$ ), within quasi harmonic approximations.

Specific heat, $C_{\mathrm{p}}$, is given by

$$
C_{\mathrm{p}}=\frac{1}{N_{\mathrm{k}}} \sum_{k, s}\left[k_{\mathrm{B}}\left(\frac{\hbar \omega_{k s}}{k_{\mathrm{B}} T}\right)^{2} \frac{e^{\frac{\hbar \omega_{k s}}{k_{\mathrm{B}} T}}}{\left(e^{\frac{\hbar \omega_{k s}}{k_{\mathrm{B}} T}}-1\right)^{2}}\right] .
$$

Thermal expansion coefficient, $\alpha$, is given by

$$
\alpha=\frac{1}{3 B} \sum_{k, s}\left[\left(-\frac{\hbar \Delta \omega_{k s}}{\Delta V}\right) \frac{\hbar \omega_{k s}}{k_{\mathrm{B}} T^{2}} \frac{e^{\frac{\hbar \omega_{k s}}{k_{\mathrm{B}} T}}}{\left(e^{\frac{\hbar \omega_{k s}}{k_{\mathrm{B}} T}}-1\right)^{2}}\right] .
$$

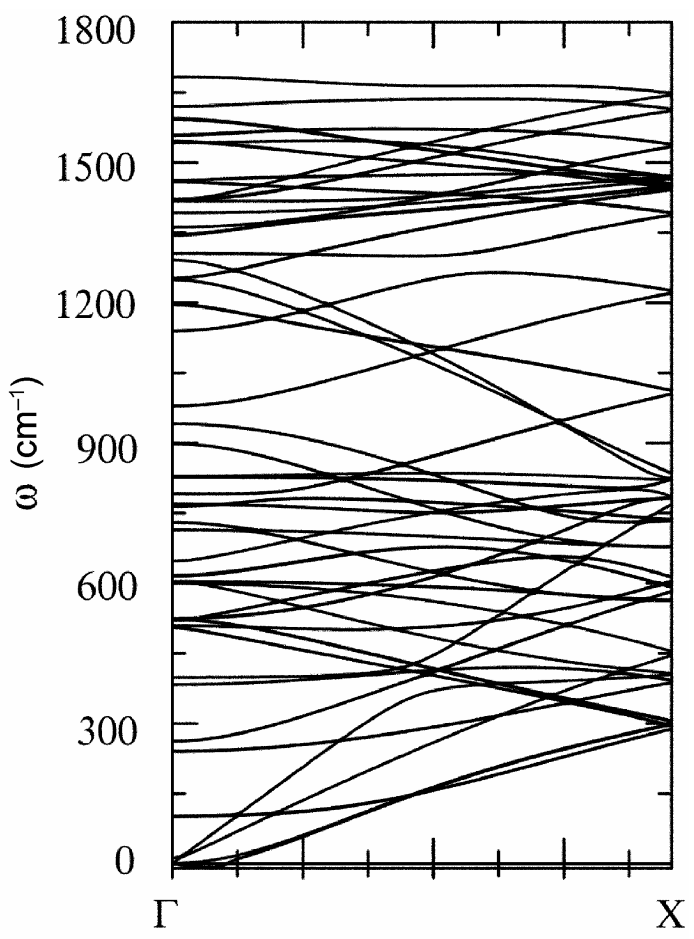

Figure 2. Phonon dispersion of isolated $(7,0)$ SWCNT: frequency, $\omega\left(\mathrm{cm}^{-1}\right)$ vs wave vector, $k\left(\mathrm{bohr}^{-1}\right)$. 


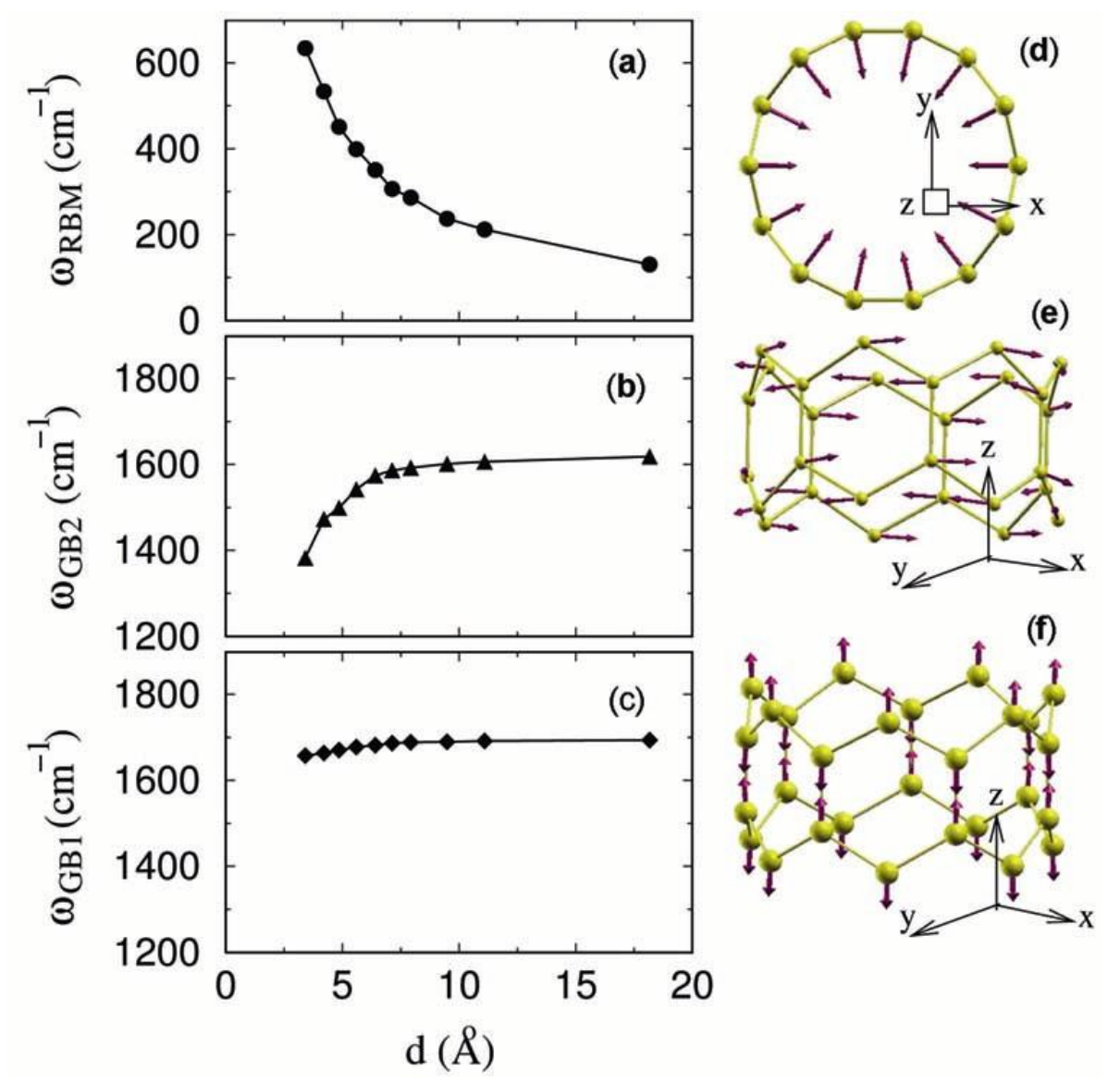

Figure 3. Frequencies of three special phonon modes (a) RBM, (b) GB2 and (c) GB1, as a function of diameter of SWCNT. Black solid points are the data points from our calculations while solid lines are the functional fit. (d), (e) and (f) show phonon eigen displacements for $(7,0)$ SWCNT corresponding to RBM, GB2 and GB1, respectively. GB1 modes are seen to be least affected due to the effect of curvature.

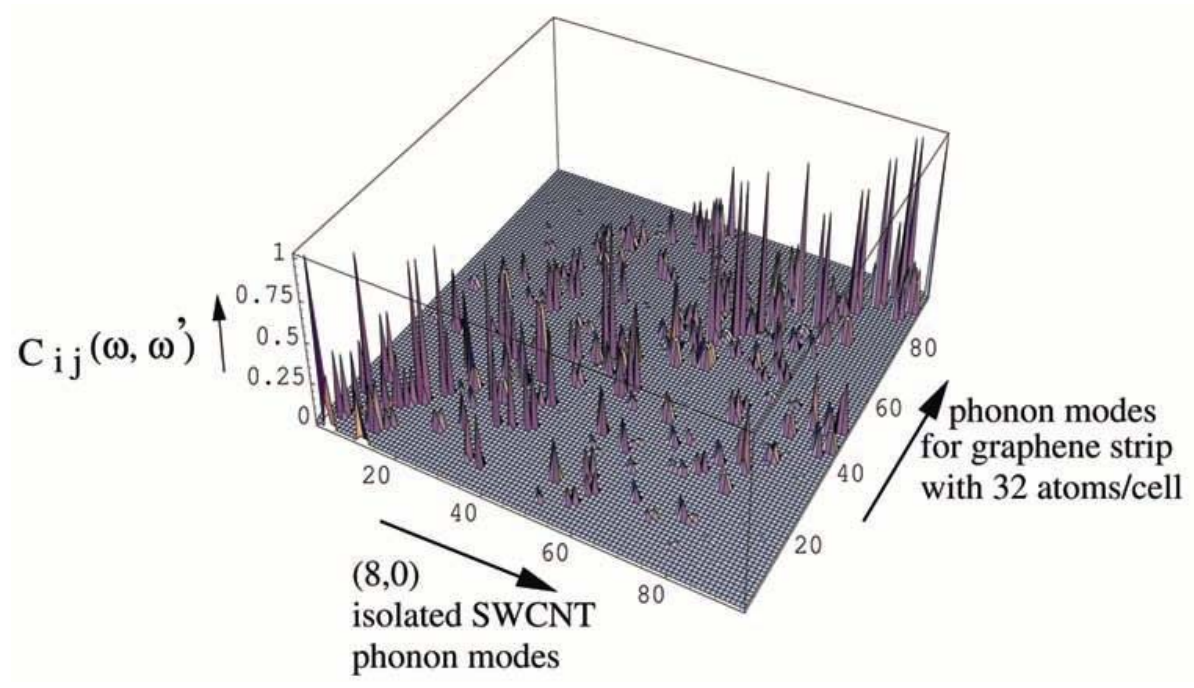

Figure 4. Correlation between phonon eigen vectors of $(8,0)$ SWCNT and that of its unrolled graphene strip form with 32 atoms/unit cell. $X$-axis displays 96 phonon eigen modes of $(8,0)$ SWCNT, $Y$-axis displays 96 phonon eigen modes of its unrolled graphene sheet form and $Z$-axis displays the normalized correlation function, $C_{i j}\left(\omega, \omega^{\prime}\right)$. 
Here, $B$ is the bulk modulus of the system and $\Delta V$ the difference between volume in equilibrium and strained configuration of a SWCNT. We note that the definition of 'radius' of the tube enters in estimation of both $B$ and $V$ and estimation of $\alpha$ does not depend on it.

To determine contributions of various phonon modes to $\alpha$, we use the mode-dependent Gruneisen parameter (GP) for a particular normal-mode $(k, s)$ given by

$$
\gamma_{k, s}=-\frac{V}{\omega_{s}(k)} \frac{\delta \omega_{s}(k)}{\delta V} .
$$

Phonon dispersions obtained for each strained configurations of an SWCNT (equilibrium, elongated and contracted along length by $1 \%$ ) are used in determining derivatives of phonon frequencies with respect to $c$, using a finite difference formula, yielding estimation for $\alpha$ and GPs.

Results for thermal expansion of a $(7,0)$ SWCNT (see figure 5) qualitatively show the $T$-dependence similar to that observed in the classical MD simulations (Kwon et al 2004) for $(10,10)$ armchair SWCNT: it is negative at low temperatures and becomes positive above $T=350 \mathrm{~K}$ (figure 5a). We find that the GP (see figure 5b) is negative for many of the lowest frequency modes and mostly positive for modes with higher frequencies. Mode-bymode contribution to $\alpha$ (see figures $5 \mathrm{c}$, d) reveals that the negative thermal expansion observed at low temperatures arises mainly from the lowest energy acoustic-like phonons, while a positive contribution from higher energy phonons dominates the thermal expansion at high tem-
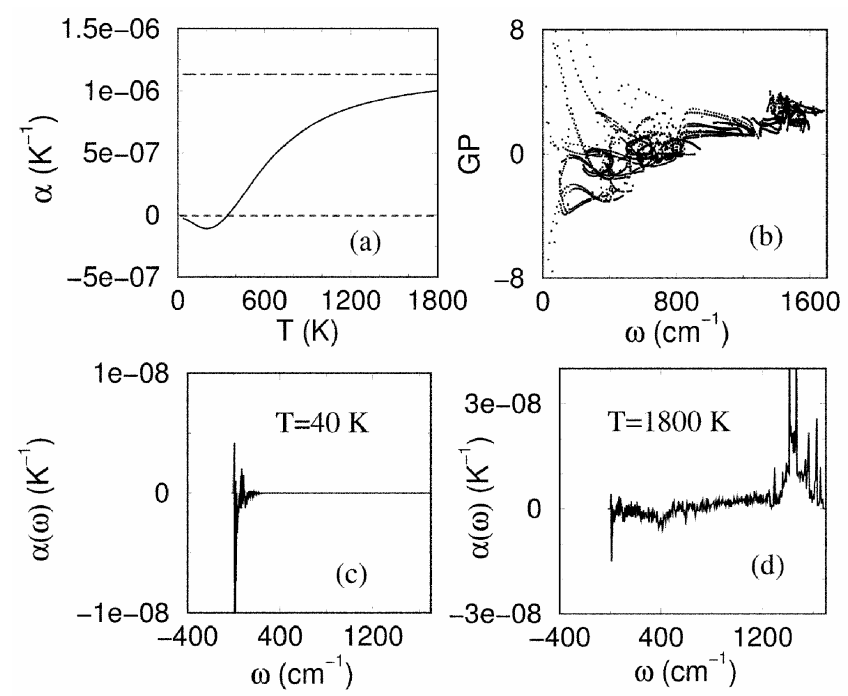

Figure 5. For isolated $(7,0)$ SWNT: (a) thermal expansion coefficient, $\alpha\left(\mathrm{K}^{-1}\right)$ vs temperature, $T(K)$. Solid line is from quantum-mechanical expression and dot-dashed line is the limit, (b) Gruneisen parameter, GP vs phonon frequency, $\omega\left(\mathrm{cm}^{-1}\right),(\mathbf{c})$ contribution of different modes to $\alpha$, i.e. $\alpha(\omega)$ vs phonon frequency, $\omega\left(\mathrm{cm}^{-1}\right)$ at $T=40 \mathrm{~K}$, and $(\mathbf{d}) \alpha(\omega)$ vs $\omega\left(\mathrm{cm}^{-1}\right)$ at $T=$ $1800 \mathrm{~K}$. peratures. Some of these very low energy modes with most prominent contribution to the negative thermal expansion exhibit a dispersion of quadratic in $k$ and their frequency varies inversely with diameter. At high temperatures, higher energy phonon modes (both acoustic and optic) with large GPs get excited (see figure 5d), leading to an overall positive $\alpha$ after a certain crossover temperature.

The main contribution to specific heat of the nanotubes comes from the vibrational part because the electronic contribution, in comparison to the vibrational contribution, is negligible above a few Kelvin. We find that the specific heat (see figure 6) of a CNT exhibits smooth monotonic increase with temperature. Slope of linear variation of $\log _{e}\left(C_{\mathrm{p}}\right)$ with $\log _{e} T$ at low temperature (see inset of figure 6 ) is constant, showing sort of a linear dependence. The high temperature classical limit of $C_{\mathrm{p}}$ (shown by dashed line in figure 6), independent of the chirality of the tube, is $3 k_{\mathrm{B}} / m \approx 2078 \mathrm{~J} / \mathrm{kg}$ Kelvin. Both the thermal expansion and specific heat results show that the classical limit is approached only at fairly high temperatures (above $1600 \mathrm{~K}$ ).

\section{Summary}

We used first-principles DFT calculations to understand structural and vibrational properties of zigzag carbon nanotubes. Nanotubes in bundled form are found to be slightly more stable (due to inter-tube interaction) than the isolated form. The radial breathing mode softens with increasing diameter: $\omega_{\mathrm{RBM}} \propto 1 / d$. Lower frequency phonon branches are greatly affected by the curvature of SWCNTs, whereas higher energy branches are relatively insensitive. Curvature effects are most prominent for SWCNTs with smaller diameter and well understood

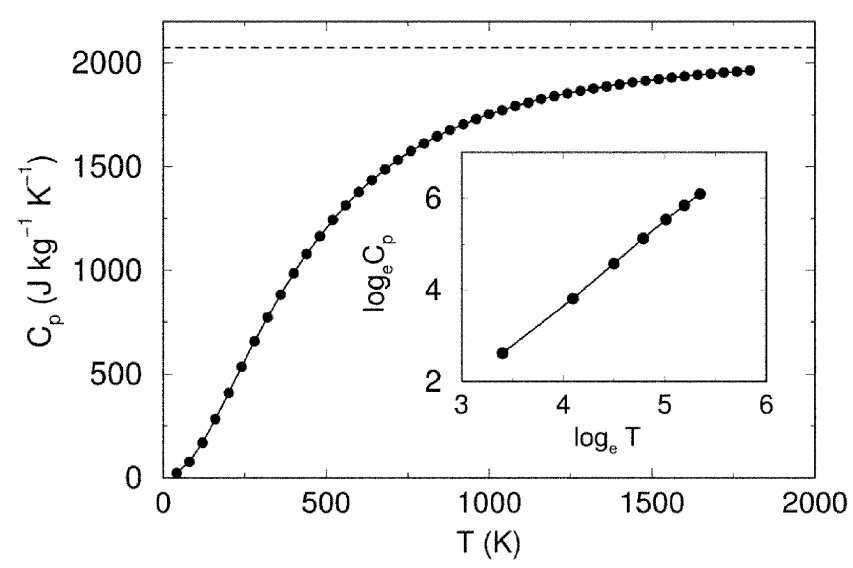

Figure 6. Specific heat, $C_{\mathrm{p}}\left(\mathrm{J} \mathrm{kg}^{-1} \mathrm{~K}^{-1}\right)$ vs temperature, $T(K)$ for $(7,0)$ isolated SWNT. Dotted line indicates the high temperature (classical) limit of $2028 \mathrm{~J} \mathrm{~kg}^{-1} \mathrm{~K}^{-1}$. Inset shows the low temperature behaviour of natural logarithm of $C_{\mathrm{p}}$. Solid circles are the calculated values. 
through SWCNTs' correlation with the normal modes of a graphene sheet. We have provided estimates of thermal expansion coefficient and specific heat of $(7,0)$ SWCNT obtained within classical and quantum treatments.

\section{References}

Bulusheva L G, Okotrub A V, Romanov D A and Tomanek D J 1998 J. Phys. Chem. A102 975

Cabria I, Mintmire J W and White C T 2003 Int. J. Quantum Chem. 9151

Hamada N, Sawada S I and Oshiyama A 1992 Phys. Rev. Lett. 681579

Ijima S 1991 Nature 35456

Jhi S-H, Louie S G and Cohen M L 2005 Phys. Rev. Lett. 95 226403

Kurti J, Kresse G and Kuzmany H 1998 Phys. Rev. B58 R8869
Kwon Y, Berber S and Tomanek D 2004 Phys. Rev. Lett. 92 15901

Lu J, Wu J, Duan W, Gu B and Johnson H T 2005 J. Appl. Phys. 97114314

Monkhorst H J and Pack J D 1976 Phys. Rev. B13 5188

Odom T W, Huang J L, Kim P and Lieber C M 2000 J. Phys. Chem. B104 2794

Ordejon P, Artacho E and Solar J M 1996 Phys. Rev. B53 R10441

Saito R, Dresselhaus G and Dresselhaus M S 1998 Physical properties of carbon nanotubes (London: Imperial College Press)

Sanchez-Portal D, Artacho E, Soler J M, Rubio A and Ordejon P 1999 Phys. Rev. B59 12678

Soler J M, Artacho E, Gale J D, Garcia A, Junquera J, Ordejon P and Sanchez-Portal D 2002 J. Phys. Condens. Matter 14 2745

Thess A et al 1996 Science 273483

Troullier N and Martins Jose Luis 1991 Phys. Rev. B43 1993 